Ultrastructural changes of liver parenchyma following digitonin-pulse perfusion of rat liver.

Quistorff, Bjørn; Rømert, P.; Matthiessen, M.E.

Published in:

Cell and Tissue Research

Publication date:

1990

Document version

Publisher's PDF, also known as Version of record

Citation for published version (APA):

Quistorff, B., Rømert, P., \& Matthiessen, M. E. (1990). Ultrastructural changes of liver parenchyma following digitonin-pulse perfusion of rat liver. Cell and Tissue Research, 261(3), 423-433. 


\title{
Ultrastructural changes of liver parenchyma following digitonin-pulse perfusion of rat liver
}

\author{
P. Romert ${ }^{1}$, M.E. Matthiessen ${ }^{2}$, and B. Quistorff ${ }^{3}$ \\ Departments of Anatomy ${ }^{1} \mathrm{C}$ and ${ }^{2} \mathrm{~A},{ }^{3}$ Department of Biochemistry A, The Panum Institute, University of Copenhagen, \\ Copenhagen, Denmark
}

Accepted May 7, 1990

Summary. It has been shown that pulse perfusion of rat liver with a digitonin-containing medium results in a highly zonated hepatocyte permeabilization, allowing selective sampling of cytosolic constituents from periportal and perivenous (centrolobular) hepatocytes "in situ". In the present paper we provide an ultrastructural evaluation of the perfusion method. Identical changes in hepatocytes from affected periportal and perivenous zones are found. Affected hepatocytes appear light (electron-lucent) in electron micrographs with a sharp transition to normal hepatocytes. The most conspicuous ultrastructural findings are: (1) transformation of the sinusoidal part of the light hepatocytes, the lipocyte processes and the endothelium of affected zones apparently unifying into a continuous layer dominated by disrupted plasma membranes and 7-nm filaments; (2) deposition of osmiophilic digitonin-cholesterol complexes along the sinusoidal plasma membranes of affected zones; and (3) reduction of the cytoplasmic matrix (cytosol) in the light hepatocytes, a dilation of the mitochondrial intermembrane space with a preserved mitochondrial matrix, and a dilation of cisternae of the granular endoplasmic reticulum. The ultrastructural findings are consistent with marker-enzyme activity measured in eluates from digitonin-perfused livers, except that lysosomes appear intact, apparently contrasting with the observed eluation of amyloglucosidase (Quistorff et al. 1985).

Key words: Hepatocytes - Liver - Ultrastructure - Digitonin perfusion - Metabolic zonation - Rat (Wistar)

It is generally accepted that the hepatocytes form a heterogeneous population of cells concerning the contents of enzymes, metabolites and several metabolic

Send offprint requests to: Paul Rømert, M.D., Department of Anatomy C, The Panum Institute, University of Copenhagen, 3, Blegdamsvej, DK-2200 Copenhagen, Denmark functions of the liver (the so-called metabolic zonation) (Sasse et al. 1975; for review, see Jungermann and Katz 1989; Quistorff 1990). Furthermore, regional differences in hepatocyte morphology have been shown in the normal (Uchiyama and Asari 1984) as well as in the diseased liver (Popper 1988). The digitonin-perfusion method was devised as a new method to study differences in enzyme activities and other parameters between periportal and perivenous hepatocytes (Quistorff et al. 1985). By applying a short digitonin pulse through the portal vein followed by a pulse via the hepatic veins, eluates from both periportal and perivenous zones of the same liver could be obtained (Quistorff and Grunnet 1987). As judged by the marker enzyme pattern in the eluates cytosolic components were eluted with negligeable contribution from the mitochondrial compartment. The permeabilizing effect of digitonin was attributed to the formation of $1: 1$ cholesterol-digitonin complexes (Zuurendonk and Tager 1974), especially in the cholesterol-rich plasma membranes. The permeabilization was accompanied by highly zone-specific cell changes as shown in a recent light-microscopic study (Quistorff and Romert 1989).

The present study describes the ultrastructural changes in periportal and perivenous hepatocytes after digitonin perfusion, primarily to provide a structural control and basis for the biochemical method, but also to describe characteristic changes after short and long perfusions with digitonin. The data are consistent with previous light-microscopic findings (Quistorff and Rømert 1989).

\section{Materials and methods}

Male Wistar rats weighing approximately $200 \mathrm{~g}$ were used. The animals were kept on a $12 \mathrm{~h}$ dark/light cycle, changing at 9 a.m. The experiments were carried out between 10 and 12 a.m. Some animals had free access to food pellets, others were fasted for $24 \mathrm{~h}$ before experimentation; all had free access to water. The rats were anesthetized with sodium pentobarbital $(400 \mu \mathrm{l}, 50 \mathrm{mg} / \mathrm{ml})$ given 
intraperitoneally. The liver was connected to the perfusion system and subsequently perfused at $35^{\circ} \mathrm{C}$ for $5-10 \mathrm{~min}$ with a buffer (Krebs and Henseleit 1932) equilibrated with $\mathrm{O}_{2} / \mathrm{CO}_{2}(19: 1)$. Thereafter a digitonin pulse (buffer with digitonin, $5 \mathrm{mg} / \mathrm{ml}$, flow rate $10 \mathrm{ml} / \mathrm{min}$ ) was applied for $8 \mathrm{~s}$ either in the direction porta $\rightarrow$ cava (portal vein $\rightarrow$ superior vena cava perfusion) or cava $\rightarrow$ porta (superior vena cava $\rightarrow$ portal vein perfusion). After the digitonin pulse the direction of flow was reversed and the liver perfused for $30 \mathrm{~s}$ with buffer without digitonin. In some experiments the digitonin pulse was extended to 15,30 and $45 \mathrm{~s}$, and also to $180 \mathrm{~s}$, which resulted in an almost completely decolorized liver, a so-called white liver (Quistorff et al. 1985). In other experiments a combined porta $\rightarrow$ cava and cava $\rightarrow$ porta digitonin perfusion (dual - digitonin - pulse perfusion) was performed with an 8-s perfusion in either direction (for further details of perfusion technique, see Quistorff and Grunnet 1987). Finally, the livers were perfused for $3 \mathrm{~min}$ using one of the following fixatives: (1) $2 \%$ formaldehyde $+1.25 \%$ glutaraldehyde in $100 \mathrm{mM}$ phosphate buffer, (2) $0.5 \%$ formaldehyde $+1 \%$ glutaraldehyde in $80 \mathrm{mM}$ phosphate buffer, and (3) $2 \%$ glutaraldehyde in $80 \mathrm{mM}$ phosphate buffer. The osmolarities were (1) $1030 \mathrm{mM}$, (2) $450 \mathrm{mM}$, and (3) $410 \mathrm{mM}$; pH 7.2; $4^{\circ} \mathrm{C}$. After perfusion fixation 1-2-mm thick slices were cut from both superficial and deep parts of all lobes and immersed in the respective fixatives for $2-3 \mathrm{~h}$. After a short rinse the slices were postfixed in $1 \%$ or $2 \% \mathrm{OsO}_{4}$ in either $100 \mathrm{mM}$ phosphate or cacodylate buffer for $2 \mathrm{~h}$. The slices were dehydrated in ethanol, transferred to propylene oxide, and embedded in Epon 812. 2- $\mu \mathrm{m}$ thick sections were cut, stained with toluidine blue, and photographed using a Leitz Orthoplan photomicroscope. For electron microscopy thin sections were cut, contrasted with uranyl acetate and lead citrate, and examined in a JEOL $100 \mathrm{CX}$ electron microscope. The criteria for quality of preservation were in accordance with previous studies on preparation of liver tissue (Rømert and Matthiessen 1975, 1979, 1981).

\section{Results}

In a recent light-microscopic study (Quistorff and Rømert 1989) we described the alterations in hepatocytes following digitonin perfusion of the liver. In brief, the hepatocytes of the affected light zones (Fig. 1) exhibited a light cytoplasm with distinct mitochondria and lysosomes. The lipid content resembled that of control (normal) hepatocytes, while the amount of glycogen was reduced. The surface of the hepatocytes facing the sinusoid was changed into a thick velvet-like structure.

Electron microscopy. reveals identical changes in hepatocytes from affected periportal and perivenous (centrolobular) zones, independent of the applied perfusion method. Affected cells appear light (electron-lucent) (Fig. 2) in contrast to the neighbouring dark (normal) hepatocytes and exhibit the following characteristic features.

Effect on the sinusoidal surface of the hepatocyte. After digitonin treatment this area, including the microvilli, forms a distinct layer with disrupted plasma membranes, 7-nm filaments and vesicles (Fig. $3 \mathrm{~A}$ and B). The structural organization is disrupted and there is no distinct perisinusoidal space. The layer also includes remnants of lipocyte processes and endothelium (Fig. 3 B). In sections not stained with uranyl acetate and lead citrate the plasma membranes exhibit distinct darkly stained deposits, which probably represent osmium-stained digitonin-cholesterol complexes. The deposits appear as dense lines separated by a light layer which is approximately $3 \mathrm{~nm}$ thick. The thickness of the dense lines varies along their length often forming regularly and symmetrically arranged thickenings. The overall thickness of the dense lines and the light layer vary from 10 to $20 \mathrm{~nm}$. Vesicles do not exhibit deposits (Fig. 3C). The sinusoidal layer is continuous along the sinusoid, and especially after prolonged digitonin pulses it forms a distinct structure detached from the underlying parts of the hepatocytes. In the light microscope the loosened structure appears to form irregular thread-like elements indicating the contours of the sinusoids (perisinusoidal structures) separated from the central (endoplasmic) portions of the hepatocytes (Fig. 4A). With the electron microscope the light-microscopic appearance was substantiated and an increasing disorganization was observed after prolonged digitonin pulses (Fig. 4B).

Effect on central portions of the hepatocytes. This part forms an entity with organelles and inclusions and a network of filaments and microtubuli. Except for liberation of glycogen particles the overall organization appears remarkably intact, even though the sinusoidal part of the hepatocyte is damaged as described above. The various components of the central part are changed in the following ways.

The amount of cytoplasmic matrix (cytosol) is considerably reduced, reflecting the elution of cytosolic macromolecules. The reduction is uniform throughout the affected hepatocyte.

The mitochondria appear slightly swollen and show a dilation of the space between the outer and inner membranes and a slight vesiculation of some of the cristae. The inner membrane usually encloses the mitochondrial matrix, which is dense, but has an altered, often bizarre shape (Figs. $3 \mathrm{~A}, 5$ ). These changes become much more pronounced upon longer exposures to digitonin (Fig. 4B). No digitonin-cholesterol complexes were deposited in the mitochondria.

The peroxisomes show a slight dilation and thinning of matrix, but otherwise appear unaffected (Figs. 5, 6). The lysosomes are not altered (Fig. 6). The tubules and cisternae of the smooth and granular endoplasmic reticulum are dilated, the latter with a partial loss of ribosomes (Figs. 5, 6). The cisternae of Golgi complexes are often also dilated. In livers from fed rats with a high glycogen content the glycogen is partly washed out, while the increased number of lipid droplets in fasted rats seems unaffected. This is qualitatively in accordance with the measurements on eluates, which showed a liberation of glycogen and triglyceride (Quistorff and Grunnet 1987). The nucleus shows a light chromatin pattern with a distinct nucleolus. The perinuclear cisterna is slightly dilated.

General effects on the liver tissue. The above-described changes in the light hepatocyte are identical throughout the cell devoid of intracellular transitions (Figs. 2, 3A). This also applies to light hepatocytes when only the sinusoidal plasma membrane on one side is affected. Few hepatocytes show changes on one sinusoidal side only. 


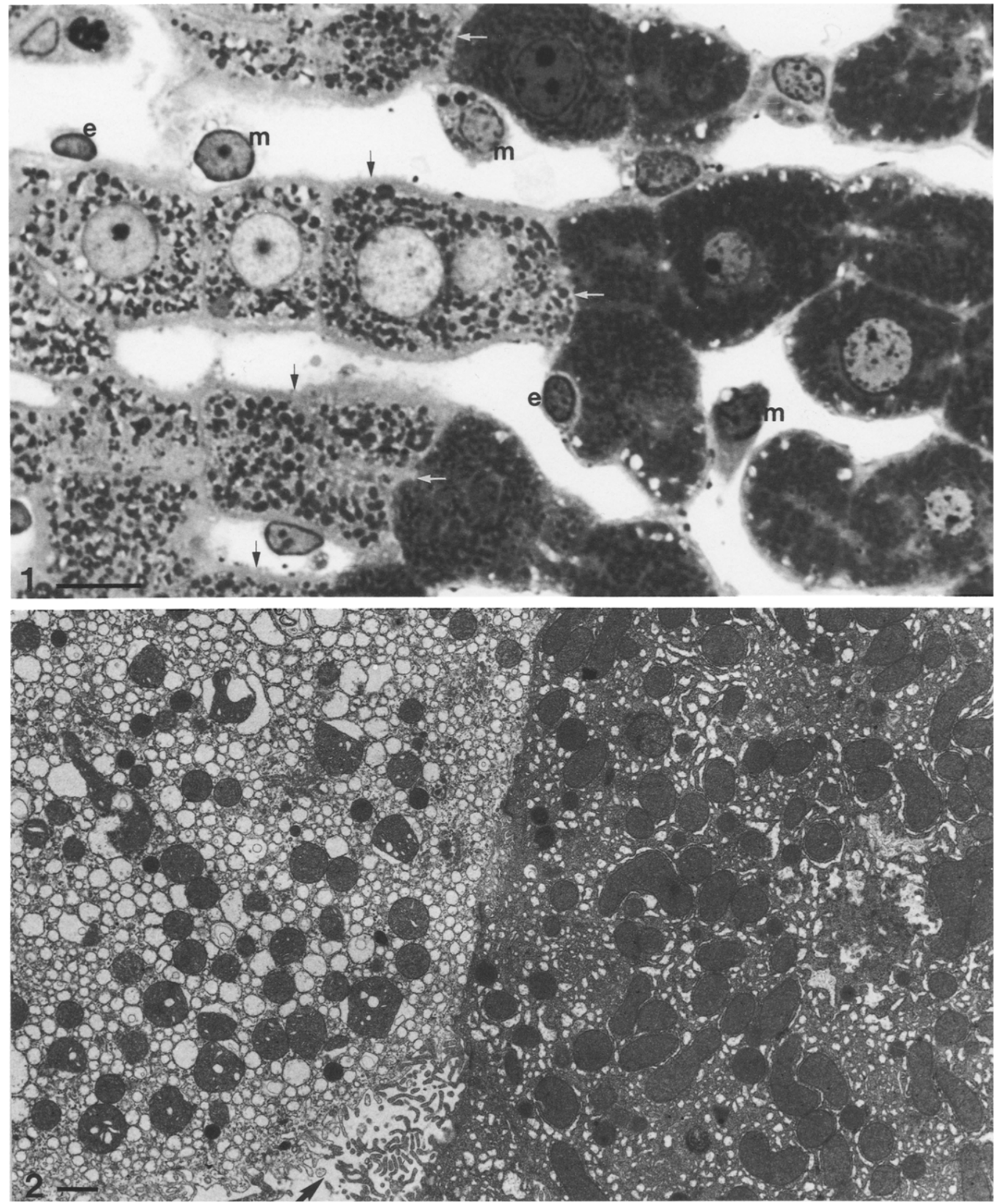

Fig. 1. Light micrograph of a section from a porta $\rightarrow$ cava perfused liver $(8 \mathrm{~s})$. Affected hepatocytes (left) contain light cytoplasm with distinct mitochondria and lysosomes. The sinusoidal surfaces appear velvet-like (black arrows). The transition from affected to normal hepatocytes is sharp (white arrows). Note that normal and damaged macrophages $(m)$ and endothelial cells $(e)$ are found alongside normal and damaged hepatocytes. Bar: $10 \mu \mathrm{m} . \times 1540$
Fig. 2. Electron micrograph showing an affected light hepatocyte (left) with a distinct borderline towards a dark hepatocyte (right). Note the abrupt change in the sinusoidal lining (arrow). Cava $\rightarrow$ porta perfusion $(8 \mathrm{~s})$. Bar: $1 \mu \mathrm{m}$. $\times 7300$ 

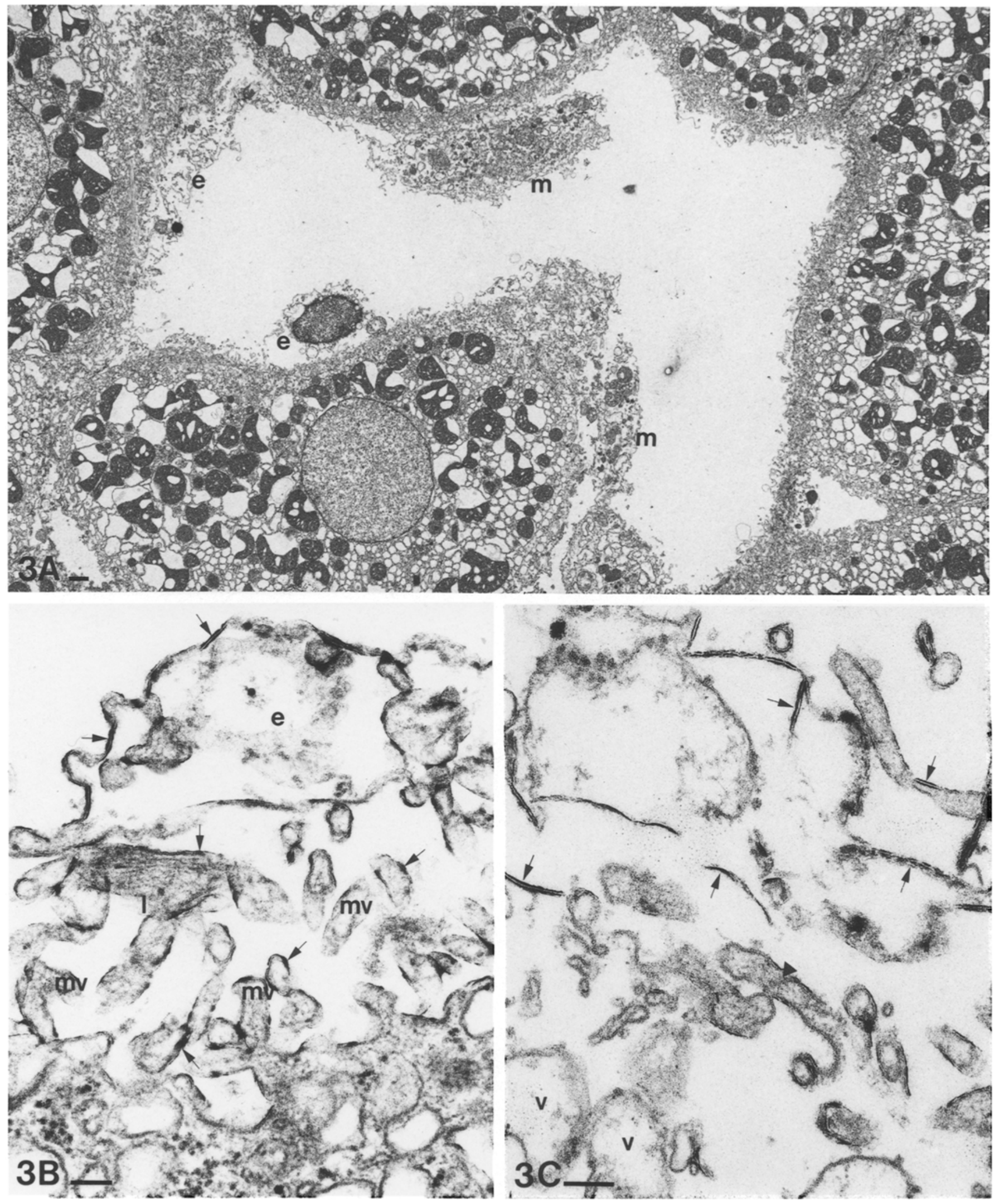

Fig. 3A-C. A Electron micrograph showing a sinusoid lined by affected light hepatocytes. The sinusoidal surface of the hepatocytes forms a distinct layer also including remnants of an endothelial cell $(e)$. In the lumen damaged mactophages $(m)$. Porta $\rightarrow$ cava perfusion $(8 \mathrm{~s})$. Bar: $1 \mu \mathrm{m}$. $\times 3640$. B Sinusoidal layer with remnants of an endothelial cell $(e)$, a lipocyte process $(l)$, and hepatocyte microvilli $(m v)$. Note osmium-stained digitonin-cholesterol

complexes along the plasma membranes (arrows). Cava $\rightarrow$ porta perfusion $(8 \mathrm{~s})$. Bar: $0.1 \mu \mathrm{m} . \times 72800$. C Osmium-stained digitonin-cholesterol complexes after digitonin perfusion (porta $\rightarrow$ cava) for $3 \mathrm{~min}$. The deposits form double-contoured lines along plasma membranes, often with a zipper-like appearance (arrows). Note that vesicles $(v)$ do not show deposits. Arrowhead 7-nm filaments. Bar: $0.1 \mu \mathrm{m} . \times 92400$ 


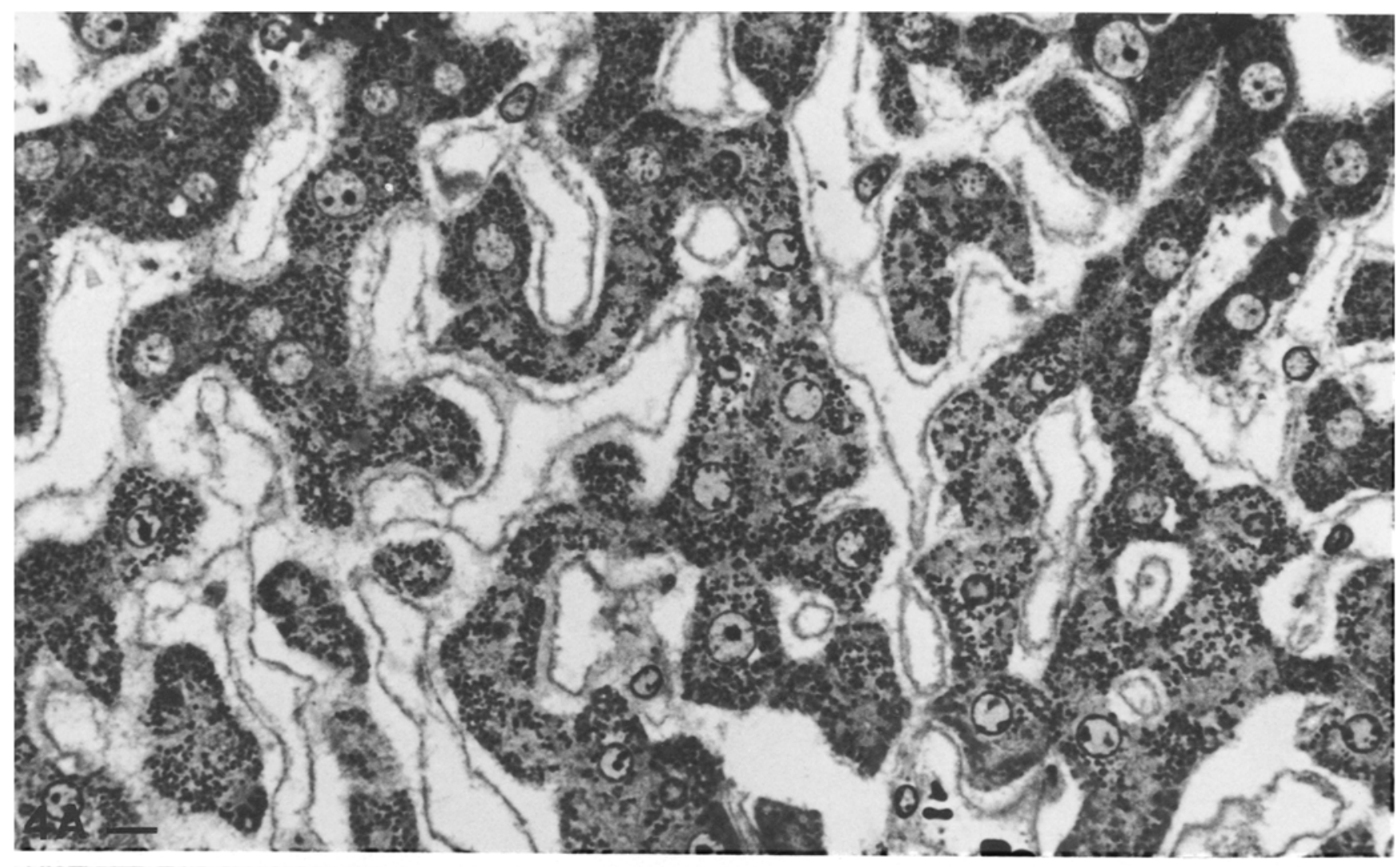

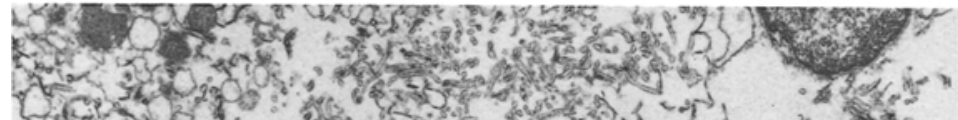

(n)

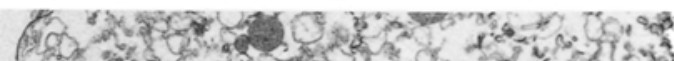

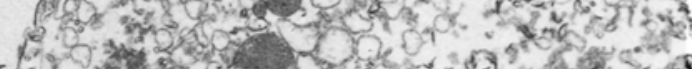
की

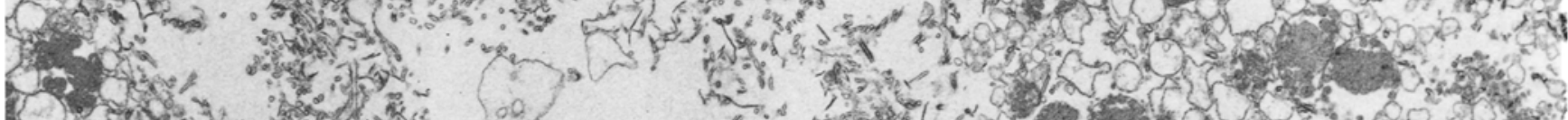

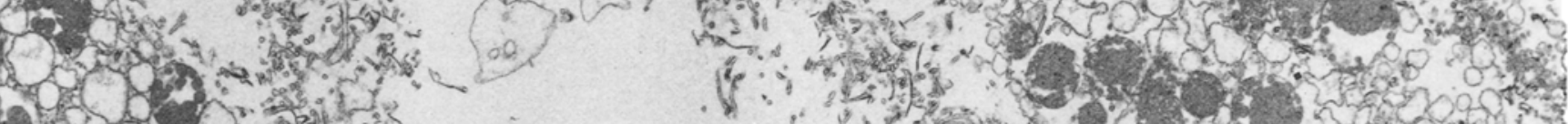

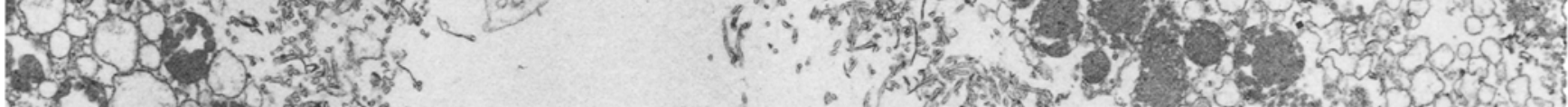
sing

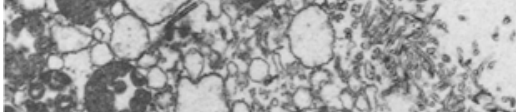

2. 0 .

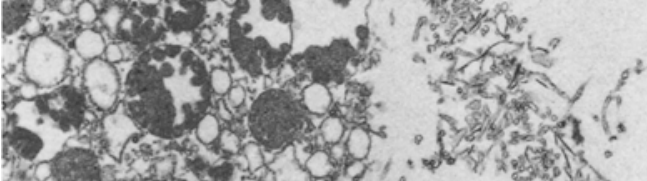

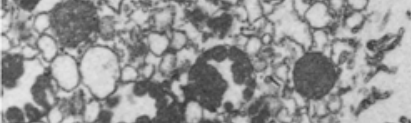

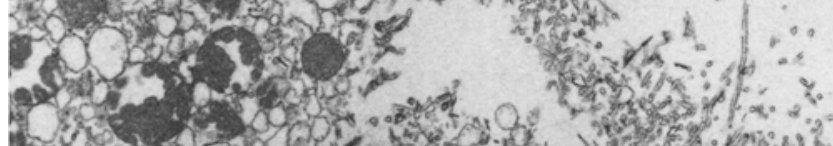

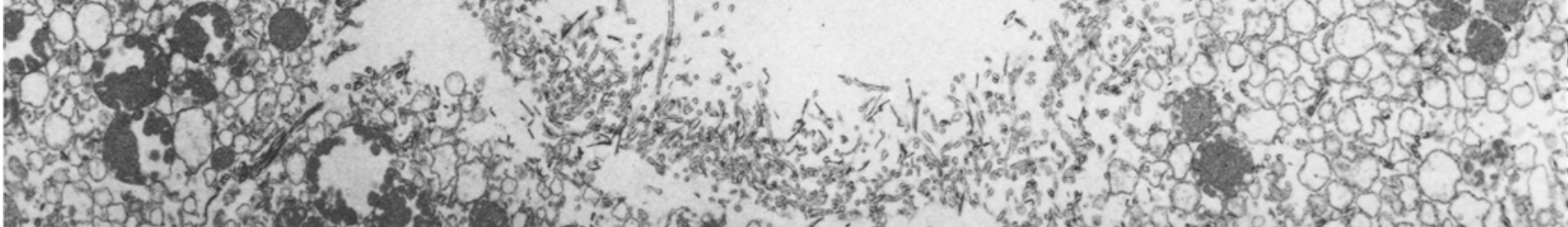

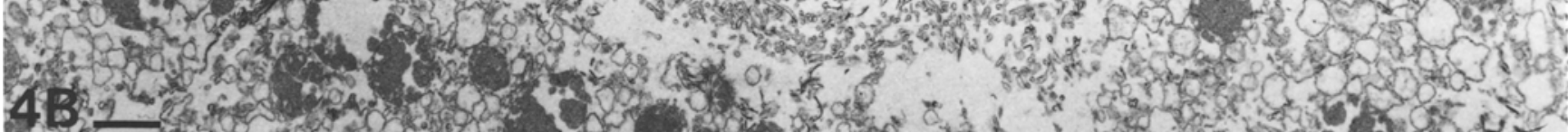
$4 \mathrm{Bg}^{4}$.

Fig. 4A-B. A Light micrograph exhibiting a velvet-like layer mostly loosened from the underlying "nude" (affected) hepatocytes. Porta $\rightarrow$ cava perfusion $(15 \mathrm{~s})$. Bar: $10 \mu \mathrm{m} . \times 615$. B Electron micrograph showing that the layer consists mostly of disorganized microvilli. Porta $\rightarrow$ cava perfusion (180 s). Bar: $1 \mu \mathrm{m} . \times 7280$ 

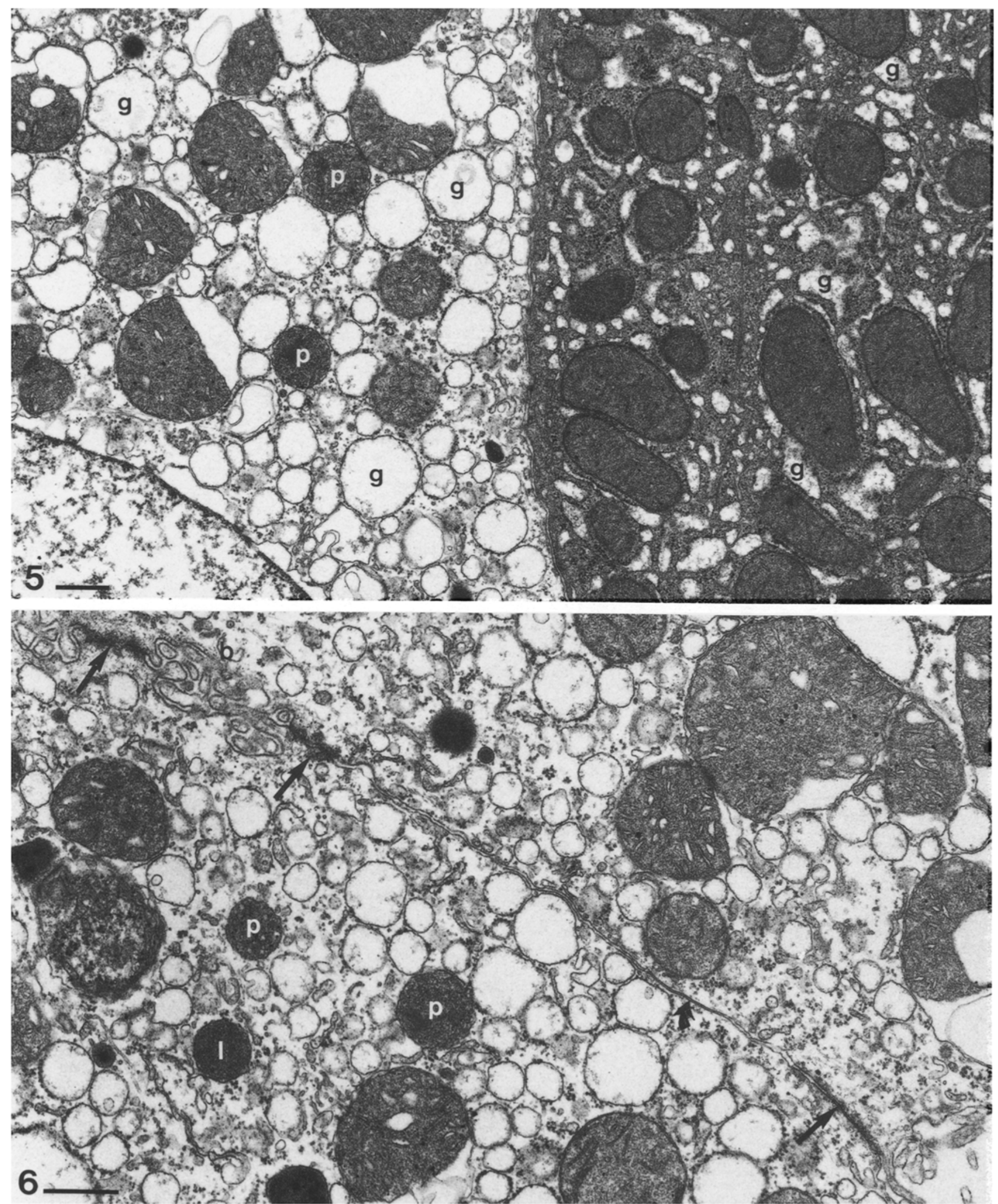

Fig. 5. Electron micrograph of a light hepatocyte and a neighbouring transitional hepatocyte. The light hepatocyte shows mitochondria with dilation of the space between outer and inner membranes, and a slight vesiculation of cristae. The granular endoplasmic cisternae $(g)$ are dilated and the cytoplasmic matrix reduced. Note intact peroxisomes $(p)$. The transitional hepatocyte has a normal morphology except for slightly dilated granular endoplasmic cisternae endowed with preserved content $(g)$. The intercellular space appears intact. Cava $\rightarrow$ porta perfusion $(8 \mathrm{~s})$. Bar: $0.5 \mu \mathrm{m} . \times 18480$
Fig. 6. Electron micrograph of two neighbouring light hepatocytes. The apparently intact intercellular space with intact plasma membranes displays tight junctions (straight arrows), a gap junction (curved arrow), and a well-preserved biliary capillary $(b)$. Note apparently intact peroxisomes $(p)$ and lysosome $(l)$. Cava $\rightarrow$ porta perfusion $(8 \mathrm{~s})$. Bar : $0.5 \mu \mathrm{m} . \times 26400$ 
The majority, however, is affected on both sides, which may be taken as an indication of a highly homogeneous perfusion. The transition from the light hepatocyte to the normal dark hepatocyte is sharp, typically with only one interposed transitional cell. The latter exhibits a slight dilution of the cytoplasmic matrix and a moderate dilation of the endoplasmic cisternae, but normal mitochondria (Fig. 5). The lateral plasma membranes (including tight junctions and gap junctions) and the canalicular membranes between even light hepatocytes seem mostly unaffected when short-time digitonin perfusions were applied (Fig. 6).

After long perfusions with digitonin (180 s) the entire liver aquires a white appearance (Quistorff et al. 1985). Under these conditions the above-described changes in the light hepatocytes are accentuated even if the overall organization is still remarkably intact (Fig. 4B). There is an increased reduction of the cytoplasmic matrix, an increased disfiguration of the mitochondria (but still with preserved dense matrix), and also a dilution of other organelles and inclusions. However, most organelles are retained in the central part of the hepatocytes. In the cytoplasm and along the lateral and canalicular intercellular plasma membranes scattered ring- and tubule-shaped deposits of digitonin-cholesterol complexes are seen.

When the normal (dark) hepatocytes of digitoninperfused livers are compared with those of controls the ultrastructure is identical.

When the osmolarity of the fixative is changed, the shape and size of the light cells are unaffected in contrast to the normal hepatocytes, which react to an increased osmolarity with shrinkage.

Macrophages, endothelial cells (Fig. 3A) and fatstoring cells show changes rather similar to those described above for hepatocytes. The changes occur in concert with those of the hepatocytes, i.e. normal macrophages are found alongside normal hepatocytes, and damaged macrophages are found alongside damaged (light) hepatocytes (Fig. 1).

The endothelium of branches of the portal and hepatic veins is affected by digitonin treatment, whereas the smooth muscle layer (and basement membrane) seems to form a protective barrier against digitonin since signs of alterations within or external to these layers are not found.

\section{Discussion}

The permeabilizing effect of digitonin was used in the digitonin-perfusion method to study eluates from periportal and perivenous hepatocytes. The effect of digitonin was attributed to a permeabilization of the hepatocyte plasma membranes caused by formation of cholesteroldigitonin complexes. Since these complexes are strongly osmiophilic and survive embedding, digitonin has also been used in ultrastructural studies to localize cholesterol in membranes (Ökrös 1968; Severs et al. 1981). In the present study the structural changes in the plasma membranes lining the perisinusoidal space reflect the permeabilizing effect of digitonin, although the precise mechanism is not understood. The linear double-contoured and strongly osmiophilic deposits observed along the sinusoidal wall of the light hepatocytes are interpreted as digitonin-cholesterol complexes formed in these locations due to the high content of cholesterol in plasma membranes (de Duve 1971). Furthermore, the dimensions of the osmium-stained linear deposits harmonize with the idea that they represent digitonin bound to the cholesterol of the plasma membranes.

It is remarkable that the transition from affected to normal hepatocytes is so sharp with only a few intervening transitional cells. This is understandable only if the digitonin perfusion in fact functions as a titration of the plasma membrane cholesterol along the perisinusoidal space as suggested previously (Quistorff et al. 1985). The lateral and canalicular cell membranes between affected hepatocytes do not seem to be affected to the same extent, when brief pulses of digitonin are used (Fig. 6). Only after 180-s digitonin perfusion are deposits clearly seen not as linear structures, but as tubules and rings along membranes. This is partly in disagreement with Kremmer et al. (1976) who showed that the highest content of unesterified cholesterol in hepatic plasma membrane subfractions was associated with the canalicular plasma membrane.

The reduction of the cytoplasmic matrix agrees with the finding that eluates show a high activity of cytosolic enzymes such as lactate dehydrogenase, alanine aminotransferase, glucokinase, and pyruvate kinase (Quistorff et al. 1985; Quistorff and Grunnet 1987). The uniform loss of matrix throughout the affected hepatocytes seems to indicate that there are no intracellular diffusion/convection barriers in the cytosolic compartment of the hepatocyte. Furthermore, the similarity of the lesion in affected periportal and perivenous hepatocytes supports the conclusion advanced previously, that the digitoninperfusion technique yields representative eluate samples from the two zones (Quistorff and Grunnet 1987).

The morphological changes seen in mitochondria are in accordance with the finding of very low activities of mitochondrial matrix enzymes, such as glutamate dehydrogenase and citrate synthase in the eluates under the present experimental conditions, and with the finding of an elution profile of the intermembrane enzyme myokinase (adenylate kinase) with a maximum between those of lactate dehydrogenase and glutamate dehydrogenase (Quistorff et al. 1985). The observed dilation of the intermembrane space and a largely preserved inner membrane and matrix correlate with the finding by Schnaitman and Greenawalt (1968) that the inner, but not the outer mitochondrial membrane is insensitive to digitonin. On the other hand, Severs et al. (1981) found that both mitochondrial membranes were completely insensitive to digitonin treatment. The fractions used by Schnaitman and Greenawalt (1968) to evaluate the localization of enzymes in isolated digitonin-treated mitochondria showed an electron-microscopic morphology of the inner membrane-matrix fraction very similar to that in the present study with an intact inner membrane and a dense matrix. The alteration in mitochondria (out- 
er membrane) is delayed (transitional cell) compared with changes in the cytosol, which agrees with the finding that eluation of myokinase localized in the intermembrane space is slower than that of cytosol enzymes (Quistorff et al. 1985).

The electron-microscopic observations are consistent with previous light-microscopic results (Quistorff and Rømert 1989) and thus support the conclusion that eluates originate from the light zones as seen with the naked eye on the liver surface (Quistorff et al. 1985; Quistorff and Rømert 1989).

The observation that the light cells do not react to a rise in fixative tonicity with a shrinkage (which normal cells do) shows that the osmotic barrier to the fixative solution is no longer present. The dilation of endoplasmic cisternae, perinuclear space and the intermembrane space of mitochondria may be caused by altered osmotic or ionic conditions following the altered properties of the plasma membrane rather than being a direct effect of digitonin. The same may account for the slight dilution of peroxisomes and nuclei. Lysosomes are surprisingly intact in spite of the fact that they usually are described as having cholesterol-rich and digitonin-sensitive membranes (Elias et al. 1978), and in spite of the fact that amyloglucosidase can be eluted (Quistorff et al. 1985; Quistorff and Grunnet 1987). It is also noteworthy that osmium-stained digitonin-cholesterol complexes are not seen in relation to the membranes of the abovementioned organelles.

The characteristic change after digitonin treatment of the sinusoidal side of the hepatocytes, the lipocyte processes and the endothelium into a distinct layer continuous along the length of the sinusoid, may indicate that these structures form an entity also in intact tissue. The content of filaments may suggest a contractile and supportive function in intact sinusoidal walls. The loosening of this layer from the remainder of the hepatocyte after longer digitonin perfusions leaves the cell in a "nude" state retaining most of the organelles and inclusions, which seem to be interwoven in a network of filaments and microtubuli. A similar phenomenon was described by Katz and Wals (1985) who removed the plasma membrane from isolated rat hepatocytes by digitonin treatment. Thus, the digitonin-perfusion technique may also prove useful as a method to study the cytostructure and the cytoskeleton of the "in situ" hepatocyte.

Acknowledgements. The authors wish to thank Ms. Marianne Juhl Christensen, Ms. Kirsten Pedersen, Ms. Lissi Immerdal, and Ms. Lis Sharwany for skilfull technical assistance.

\section{References}

Duve C de (1971) Tissue fractionation. Past and present. J Cell Biol 50:20D-55D

Elias PM, Goerke J, Friend DS (1978) Freeze-fracture identification of sterol-digitonin complexes in cell and liposome membranes. J Cell Biol 78:577-596

Jungermann K, Katz N (1989) Functional specialization of different hepatocyte populations. Physiol Rev 69:708-764

Katz J, Wals PA (1985) Studies with digitonin-treated rat hepatocytes (nude cells). J Cell Biochem 28:207-228

Krebs HA, Henseleit K (1932) Untersuchungen über die Harnstoffbildung in Tierkörpern. Hoppe-Seyler's Z Physiol Chem $210: 33-66$

Kremmer T, Wisher MH, Evans WH (1976) The lipid composition of plasma membrane subfractions originating from the three major functional domains of the rat hepatocyte cell surface. Biochim Biophys Acta 455:655-664

Ökrös I (1968) Digitonin reaction in electron microscopy. Histochemistry 13:91-96

Popper H (1988) Hepatocellular degeneration and death. In: Arias IM, Jakoby WB, Popper H, Schachter D, Shafritz DA (eds) The liver: Biology and pathobiology, 2nd edn. Raven Press, New York, pp 1087-1103

Quistorff B (1990) Metabolic heterogeneity of liver parenchymal cells. Essays Biochem 25:83-136

Quistorff B, Grunnet N (1987) Dual-digitonin pulse perfusion: Concurrent sampling of periportal and perivenous cytosol of rat liver for determination of metabolites and enzyme activities. Biochem J 243:87-95

Quistorff B, Rømert P (1989) High zone-selectivity of cell permeabilization following digitonin-pulse-perfusion of rat liver. A reinterpretation of the microcirculatory zones. Histochemistry 92: 487-498

Quistorff B, Grunnet N, Cornell NW (1985) Digitonin perfusion of rat liver. A new approach in the study of intra-acinar and intra-cellular compartmentation in the liver. Biochem $\mathfrak{J}$ 226:289-297

Rømert P, Matthiessen ME (1975) Fixation of foetal pig liver for electron microscopy. Anat Embryol 147:243-258

Rømert P, Matthiessen ME (1979) Improved utilization of tissue blocks for electron microscopy. Acta Anat 104:388-393

Rømert P, Matthiessen ME (1981) Swelling of mitochondria in immersion-fixed liver tissue. Acta Anat 109:332-338

Sasse D, Katz N, Jungermann K (1975) Functional heterogeneity of rat liver parenchyma and isolated hepatocytes. FEBS Lett $57: 83-88$

Schnaitman C, Greenawalt JW (1968) Enzymatic properties of the inner and outer membranes of rat liver mitochondria. J Cell Biol 38:158-175

Severs NJ, Warren RC, Barnes SH (1981) Analysis of membrane structure in the transitional epithelium of rat urinary bladder. J Ultrastruct Res 77:160-188

Uchiyama Y, Asari A (1984) A morphometric study of the variations in subcellular structures of rat hepatocytes during 24 hours. Cell Tissue Res 236:305-315

Zuurendonk PF, Tager J (1974) Rapid separation of particulate components and soluble cytoplasm of isolated rat-liver cells. Biochim Biophys Acta 333:393-399 